
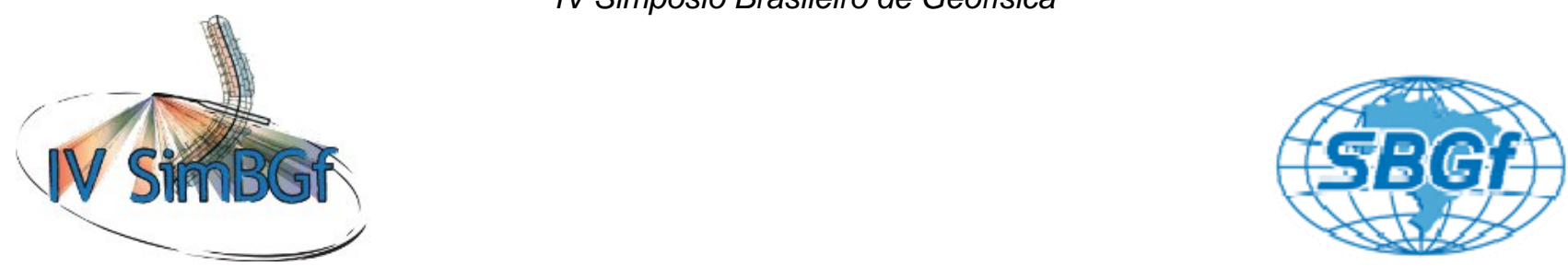

\title{
Arquitetura sedimentar e progradação dos sistemas plataformais ao largo do estado do Rio de Janeiro - bacia de Santos
}

Maia, R. M. C. $\left({ }^{*}\right)^{1}$; Reis, A.T. ${ }^{2}$; Silva, C. G. ${ }^{1} ;$ Arantes-Oliveira, R. ${ }^{2+}$; Malaquias, L. ${ }^{2++}$;

${ }^{1}$ Universidade Federal Fluminense - Lagemar, renata.ocn@gmail.com; ${ }^{2}$ Faculdade de Oceanografia-UERJ; ${ }^{+}$Bolsista PIBIC-CNPq; ${ }^{++}$Bolsista de IC-FAPERJ

Copyright 2010, SBGf - Sociedade Brasileira de Geofísica

Este texto foi preparado para a apresentação no IV Simpósio Brasileiro de Geofísica, Brasília, 14 a 17 de novembro de 2010. Seu conteúdo foi revisado pelo Comitê Técnico do IV SimBGf, mas não necessariamente representa a opinião da SBGf ou de seus associados. É proibida a reprodução total ou parcial deste material para propósitos comerciais sem prévia autorização da SBGf.

\section{Resumo}

A análise de perfis sísmicos da plataforma continental da porção nordeste da Bacia de Santos permitiu a identificação de cinco sequências sísmicas (sequências Sq1 a Sq5), sendo quatro delas progradacionais (Sq1 a Sq4). As sequências são limitadas por superfícies discordantes que sugerem processos erosivos por prolongada exposição subaérea desenvolvidos durante níveis de mar baixo (superfícies S1 a S5). As sequências sísmicas são caracterizadas por clinoformas dominantemente oblíquas, compreendendo prismas de borda de plataforma, interpretados como sequências deposicionais depositadas sob condições de regressão forçada. A calibração da interpretação sísmica com dados crono-estratigráficos possibilitou posicionar as cinco sequências (Sq1 a Sq5) nos últimos 500 kyr. A deposição destas sequências está provavelmente relacionada a ciclos de variação glacio-eustática da ordem de 100-120 kyr. A análise sísmica evidenciou uma variabilidade temporal das sequências desde a Sq1 a Sq4: as sequências mais basais (Sq1 e Sq2) preservaram a deposição mais restritas à borda da plataforma com maior deposição frontal enquanto as sequências mais superiores (Sq3 e Sq4) se estendem até áreas mais proximais e possuem o desenvolvimento de depositos frontais mais limitado. A análise sísmica também evidenciou variabilidade no sentido leste-oeste da área de estudo: na porção leste, a maioria das sequências é pobremente preservada sobre a plataforma, estando presente apenas na região de borda de plataforma com menor deposição frontal em relação ao setor oeste; já na porção oeste da área de estudo, as sequências são mais espessas e alcançam a plataforma continental média/interna, preservando sistemas deposicionais agradacionais (sistemas transgressivos ou de mar alto).

\section{Introdução}

Vários estudos de caso ao redor do globo abordam os diferentes aspectos arquiteturais das plataformas continentais quaternárias, resultantes da relação entre taxa de aporte e de acomodação sedimentar. Estudos dessa natureza, abrangendo grandes porções das plataformas continentais, são relativamente raros no Brasil, e mesmo inexistentes na plataforma continental adjacente ao Estado do Rio de Janeiro. O presente estudo focaliza a organização e as modificações do registro sedimentar da seção rasa da plataforma continental da porção nordeste da Bacia de Santos.

\section{Metodologia/ Problema Investigado}

Para a realização desse trabalho foram utilizados perfis de sísmica de reflexão rasa monocanal das Operações GEOMAR XX e GEOMAR XVI que recobrem a plataforma da porção nordeste da Bacia de Santos, desde a llha de São Sebastião até Cabo Frio (fig. 1A). Foram também utilizados curvas globais de variação eustáticas baseadas na variação da razão isotópica de $\delta^{18} \mathrm{O}$, compiladas por Rabineau et al. (2006), e dados cronoestratigráficos de poços fornecidos pelo BDEP.

\section{Resultados}

A interpretação sísmica, baseada nos conceitos de Estratigrafia de Sequências de Alta Resolução (Catuneanu, 2006), permitiu a subdivisão da seção estratigráfica mais superior da porção nordeste da bacia em 5 unidades sísmicas principais: Sq1, Sq2, Sq3, Sq4 e Sq5 (fig. 2). Cada uma dessas unidades é limitada acima e abaixo por superfícies bastante irregulares que sugerem a atuação de processos erosivos relacionados a longos períodos de exposição subaérea da plataforma continental, durante queda relativa do nível do mar (Maia et al., 2010 - superfícies S1, S2, S3, S4 e S5, fig. 2). As unidades Sq1-Sq4 são caracterizadas por um padrão deposicional dominantemente progradacional formadas essencialmente por uma sucessão de clinoformas na plataforma externa. De acordo com estas características, cada unidade foi relacionada a depósitos regressivos preservados na parte distal do sistema (fig. 2), formadas sob condições dominantes de regressão forçada (sensu Posamentier et al., 1992)(fig. 3).

O cruzamento da base de dados sísmicos com informações crono-estratigráficas de um poço na bacia de Campos, disponíveis na literatura, fornece um 


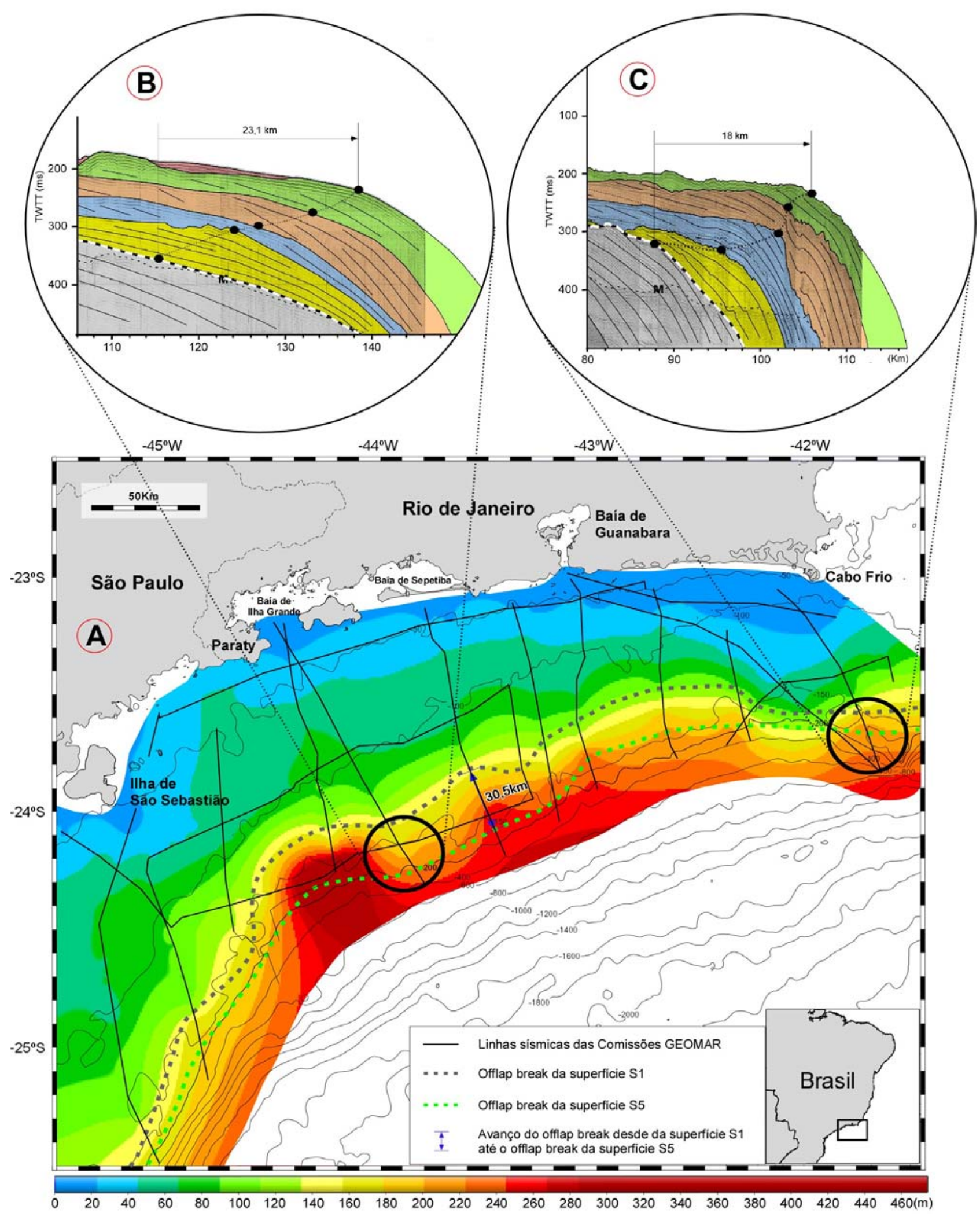

Figura 1 - Mapa de localização da perfilagem sísmica (Operações Geomar em linhas pretas finas), com o posicionamento dos offlap breaks de cada superfície relacionada às cinco sequências identificadas. 


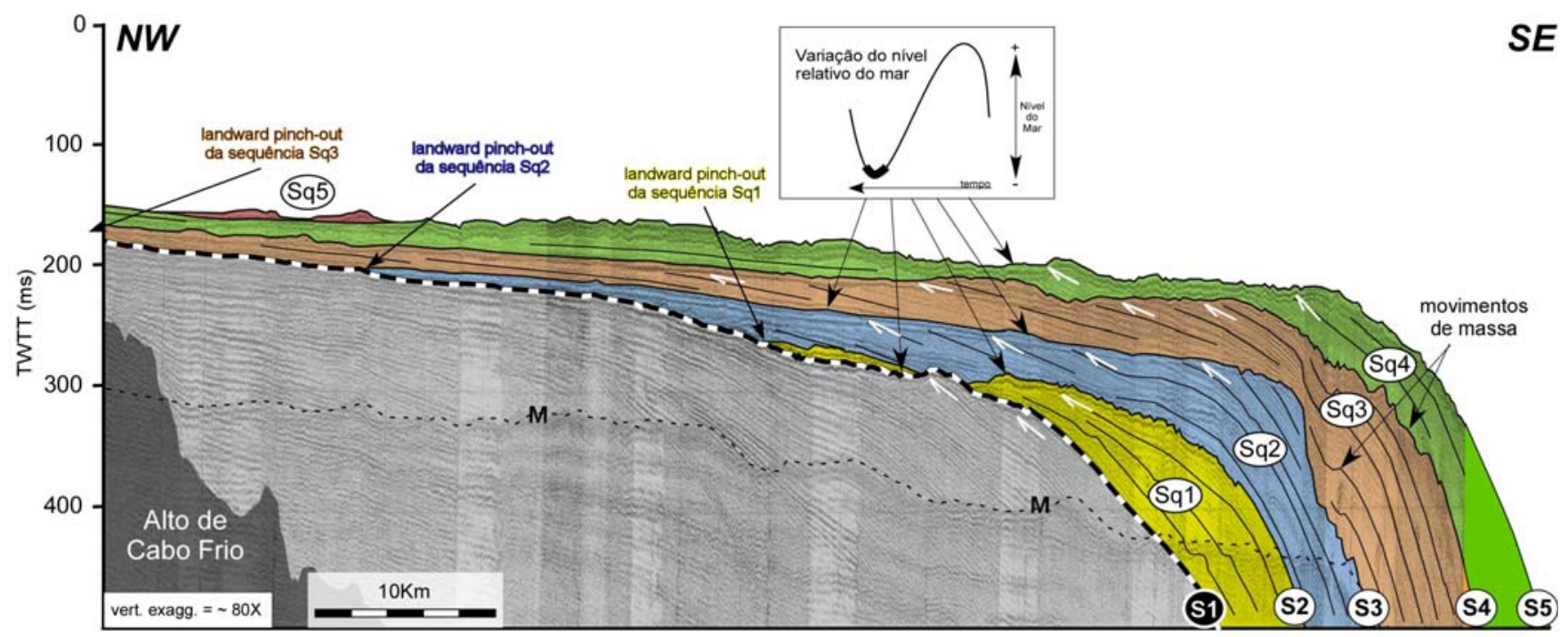

Figura 2 -Perfil sísmico ilustrando as superfícies e as sequências sísmicas interpretadas neste estudo. Localização na figura 1.

possível limite temporal para a superfície-base dessas 5 unidades sismicas identificadas (discordância S1) indicando que essa discordância é correlacionável a uma superfície regional da plataforma continental, datada na Bacia de Campos em cerca de 500 ka (MesoPleistoceno, chamada de Marco P; Silva, 1992).

A integração dos resultados da análise sísmica, das informações crono-estratigráficas e de dados mundiais de estratigrafia isotópica (Rabineau et al., 2006) indica que essas cinco sequências deposicionais (Sq1-Sq5) empilhadas sobre a superficie $S 1(\sim 500 \mathrm{ka})$ teriam se depositado num intervalo temporal de cerca de $500 \mathrm{ka}$. Através da correlação de cada superfície com posições de níveis do mar durante máximos glaciais, foi correlacionar as 5 unidades sísmicas com sequências deposicionais desenvolvidas durante ciclos glacioeustáticos de cerca 100-120 ka de duração e de 120 m de amplitude (Clark et al., 2003) (fig. 4). A interpretacao da ciclicidade deposicional proposta acima é ainda consistente com as evidencias encontradas em outras margens Quaternarias ao redor do mundo, onde ciclos eustaticos de alta frequencia $\left(4^{\mathrm{a}}-5^{\mathrm{a}}\right.$ ordem) representam o principal controle nos ciclos deposicionais e padrões de acamamento interno (ex., Trincardi e Correggiari, 2000; Ridente e Trindcardi, 2002; Rabineau et al., 2005; Tripsanas e Piper, 2008).

A análise sísmica e o mapa de isópacas total das sequências (fig. $1 \mathrm{~A}$ ) evidenciam variabilidade lateral na sua espessura e no seu grau de preservação ao longo da área de estudos. Os sucessivos deslocamentos da posição de alcance proximal das sequências em direção ao continente (landward pinch-out, fig. 2) evidenciam que a área de preservação das sequências aumenta a partir da deposição da Sq1 (bem restrita às porções externas da plataforma) se ampliando progressivamente até o total recobrimento da plataforma, observado após a deposição da sequência Sq4 (fig. 2). Ao mesmo tempo, a espessura das sequências preservadas aumenta ao longo do tempo: as sequências mais basais (Sq1 e Sq2) possuem uma espessura menor que as sequências posteriores (Sq3 e Sq4). O aumento progressivo do grau de preservação das sequências indica que o aumento relativo de espaço de acomodação sedimentar e o afogamento progressivo da plataforma entre a deposição das sequências Sq1 e Sq4 devem ser creditados a mecanismos de subsidência, que operaram na escala do Quaternário Médio-Superior. Além disso, o mapa de isópacas total (fig. 1A) evidencia que a área de espessura e de maior preservação das sequências deposicionais Sq1-Sq4 ocorre na porção centro-oeste da plataforma, aproximadamente entre a Baia de Guanabara e a Ilha de São Sebastião. Esta configuração indica que no sentido leste-oeste, há também um aumento diferencial de criação de espaço de acomodação sedimentar durante o Quaternário Médio-Superior. A observação das linhas sísmicas também sugere uma maior criação de espaço de acomodação ao longo do setor centro oeste, onde se observam superfícies de fundo e de paleo-fundo marinho mais inclinadas quando comparadas ao setor leste da área de estudo (fig. 1B e C). Estas evidências apontam para uma subsidência mais importante na porção centro-oeste da área, ou para um maior espaço de acomodação sedimentar na área herdado de uma subsidência diferencial pretérita. Já no setor leste, a progradação da plataforma é de aproximadamente $18 \mathrm{~km}$ (fig. 1C), demonstrando que nessa porção a sedimentação foi menos significativa, ou então que a ocorrência de movimentos de massa podem ter afetado a espessura das sequências progradacionais preservadas, como observado na figura 2 . 
A)

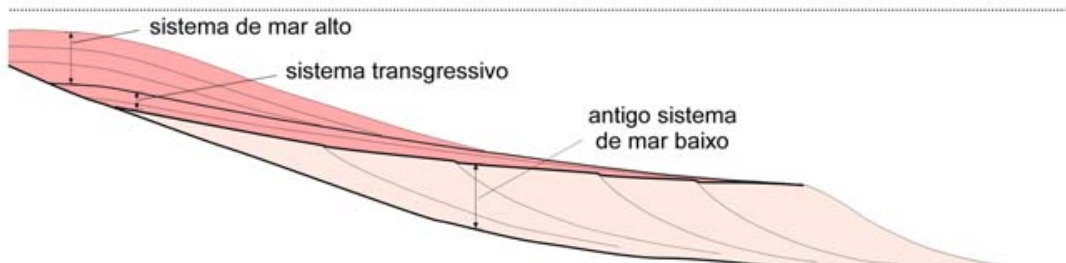
NM

Curva de variação de nivel do mar
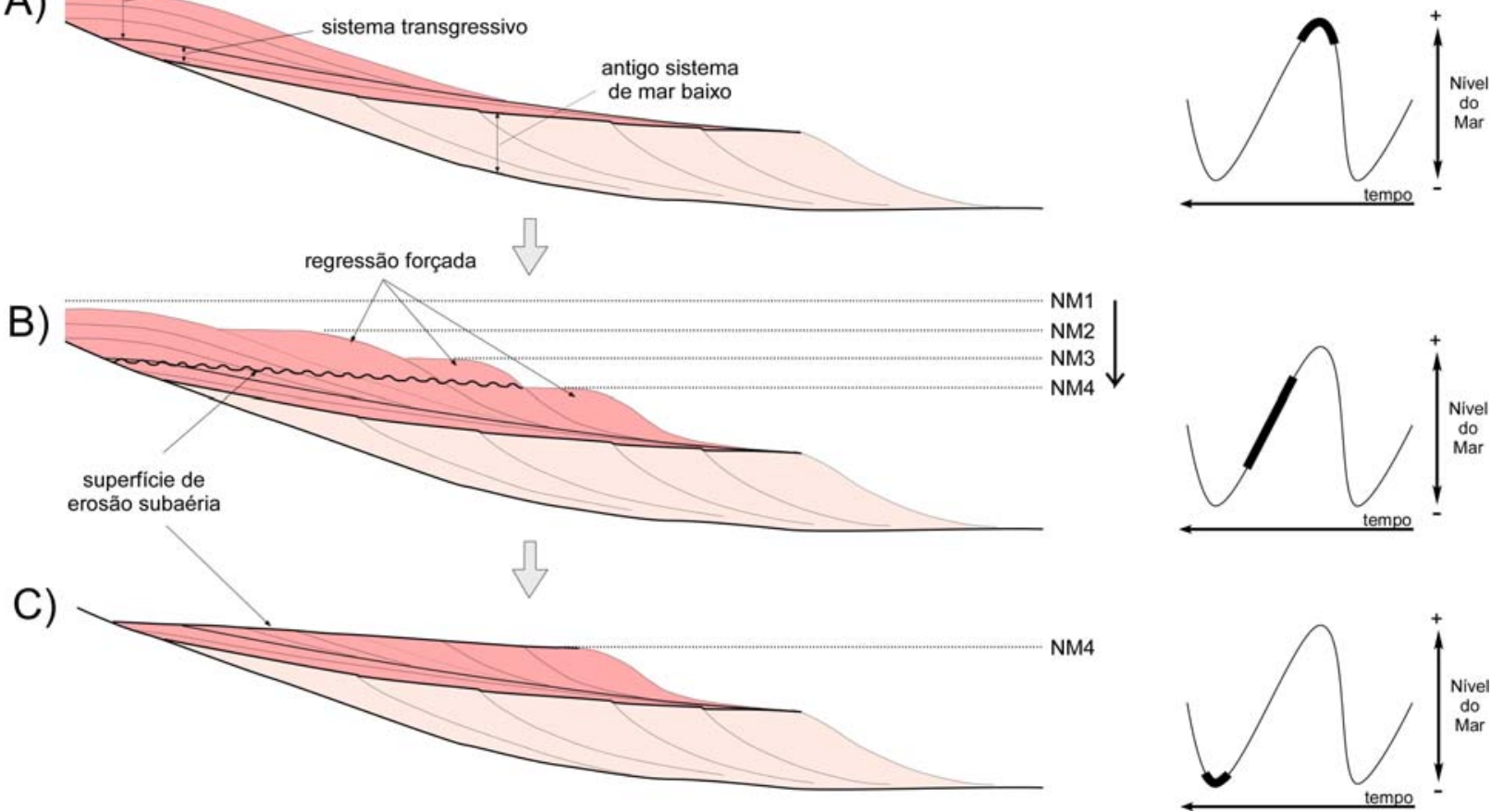

Figura 3 - Modelo esquemático da arquitetura sedimentar resultante de condições de regressão forçada (modificado de Ridente e Trincardi, 2002). A) Deposição dos sistemas sedimentares transgressivos e de mar alto; B) Rebaixamentos sucessivos do nível de base, com a formação de prismas de regressão forçada e erosão subsequente do seu topo a cada rebaixamento do nivel marinho; C) Perfil final, com os prismas regressivos truncados acima pela superfície erosiva final de exposição subaérea.

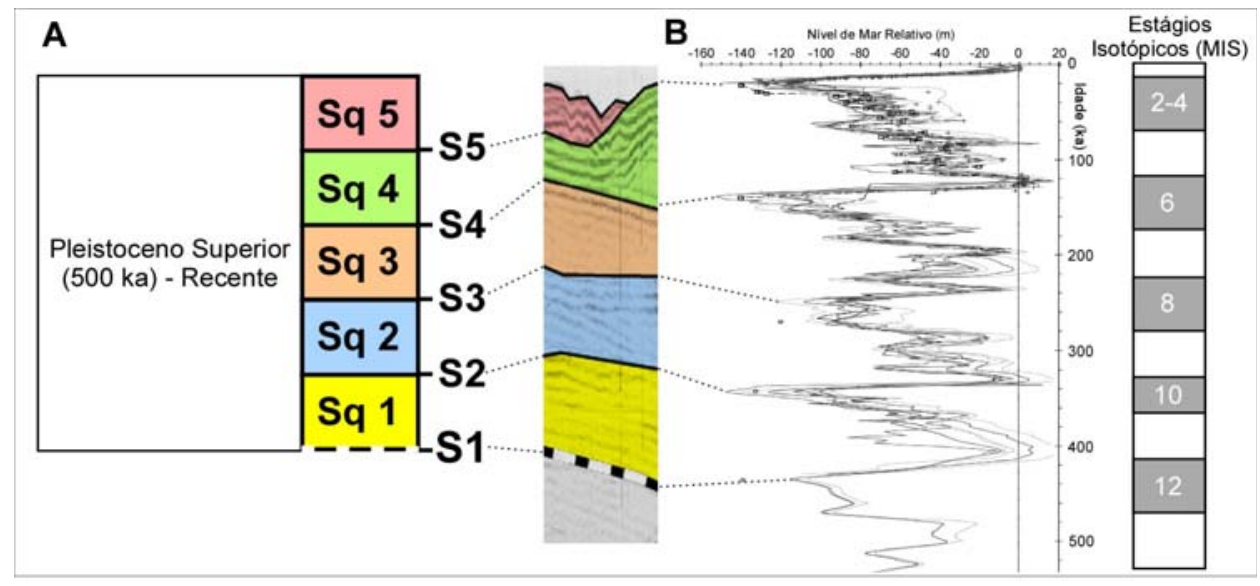

Figura 4 - A) Coluna estratigráfica da seção rasa da plataforma da área de estudos, calibrada com dados de poços. B) Proposição de ciclicidade deposicional baseada na correlação com curvas globais de variação isotópica de $\delta^{18} \mathrm{O}$, compiladas por Rabineau et al. (2006).

\section{Conclusões}

- A calibração dos dados cronoestratigráficos com a perfilagem sísmica possibilitou a correlação do Marco $P$ (idade possível de 500 ka, Silva, 1992) com a superfície $\mathrm{S} 1$, indicando que as cinco sequências $\mathrm{Sq} 1$ a $\mathrm{Sq} 5$ estão relacionadas com ciclos de variação glácio-eustática de quarta ordem (100-120 kyr, Catuneanu et al., 2006).

- Os sucessivos deslocamentos da posição dos landward pinch-out das sequências cada vez mais proximais indicam um aumento progressivo do grau de preservação das sequências, e por consequência, um aumento 
relativo de espaço de acomodação sedimentar relacionados a algum mecanismo de subsidência que operou na escala do Quaternário Médio-Superior.

- A maior inclinação do fundo e dos paleo-fundos marinhos das sequências desenvolvidades nos últimos 500 ka na porção centro-oeste, em conjunto com uma maior progradação na área, nos permitem afirmar que essa porção da plataforma apresenta um aporte sedimentar mais significativo em relação à porção leste da area de estudo.

\section{Referências}

Catuneuanu, O. 2006. Principles of Sequence Stratigraphy. Ed. Elsevier. 375p.

Clark, P.U.; Archer, D.; Pollan, D.; Blum, J. D.; Jose, A.; Brovkin, V.; Mix, A.C.; Pisias, N.G.; Martin, R. 2006. The middle Pleistocene transition: characteristics, mechanisms, and implications for long-term changes in atmospheric pCO2. Quaternary Science Reviews, v. 25, p. 3150-3184.

Maia, R. M. C.; Reis, A. T.; Alves, E. C.; Silva, C. G.; Silva, A.; Guerra, J. V.; Gorini, C.; Gorini, C.; Silva, A.; Arantes-Oliveira, R. (2010). Architecture and stratigraphic framework of shelf sedimentary systems off Rio de Janeiro state, northern Santos basin-Brazil. . Brazilian Journal of Oceanography, 58(special issue, IGCP526):15-29, 2010.

Posamentier, H. W.; Allen; P. G.; James, D. P.; Tesson, M. 1992. Forced regression in a seismic stratigraphic framework: concepts, examples and exploration. AAPG Bulletins, v. 76: 1687-1709.

Rabineau, M., Berbe, S., Aslanian, D., Olivet, J.L., Joseph, P., Guillocheau, F.,Bourrillet, J.F., Ledrezen, E., Granjean, D. (2005). Sedimentary sequences in the Gulf of Lions: a record of 100,000 years climatic change. Marine and PetroleumGeology, 22(6-7):775804.Rabineau, M., Berné S., Aslanian, D., Olivet, J-L, Joseph, P., Guillocheau, F., Bourillet, JF., Ledrezen, E., Grajeon, D. 2006. Paleo sea levels reconsidered from direct observation of paleoshoreline position during Glacial Maxima (for the last 500,000 yr). Earth and Planetary Science Letters 252 119-137.

Ridente, D., Trincardi, F. 2002. Eustatic and tectonic control on deposition and variability on Quaternary regressive sequences in the Adriatic basin (Italy). Marine Geology, 184:273-293.

Silva, A. 1992. Evolução sedimentar pós-miocênica na área nordeste da Bacia de Campos, Rio de Janeiro. 57p. Dissertação (Mestrado) - Universidade Federal do Rio de Janeiro, UFRJ, Rio de Janeiro.

Trincardi, F.; Corregiari, A. Quaternary forced-regression deposits in the Adriatic basin and the record of composite sea-level cycles. In: Seismic response to forced regression. In: HUNT, D.; GAWTHORPE, R. L. (eds). Sedimentary responses to forced regressions. Geological Society, London, Special Publications, v. 172, p. 245-269. 2000.

Tripsanas, E. K.; Piper, D. J. W. Late Quaternary stratigraphy and sedimentology of Orphan Basin: Implications for meltwater dispersal in the southern Labrador Sea. Palaeogeography, Palaeoclimatology, Palaeoecology, v, 260, n. 3-4, p. 521-539. 2008. 